\title{
Effect of microRNA-370 on coronary atherosclerosis and its underlying mechanism
}

\author{
$\mathrm{XINGE} \mathrm{SHI}^{1,2}$ and XIN CHEN ${ }^{1}$ \\ ${ }^{1}$ Department of Thoracic and Cardiovascular Surgery, Nanjing First Hospital, \\ Nanjing Medical University, Nanjing, Jiangsu 210006; ${ }^{2}$ Department of Cardiovascular Surgery, \\ First Affiliated Hospital of Henan University of Science and Technology, Luoyang, Henan 471003, P.R. China
}

Received April 5, 2018; Accepted September 13, 2018

DOI: $10.3892 /$ etm.2018.6961

\begin{abstract}
As a global health problem, cardiovascular disease threatens the lives of human beings. It has been reported that microRNAs (miRs) are important in regulating coronary atherosclerosis. In the present study, the expression levels of miR-370 in peripheral blood mononuclear cells of patients with coronary atherosclerosis were significantly increased compared with healthy patients, as demonstrated by reverse transcription-quantitative polymerase chain reaction analysis. Additionally, the target of miR-370 was predicted as Forkhead Box 1 (FOXO1) with bioinformatics, and was confirmed by a dual luciferase assay. The mRNA and protein expression levels of FOXO1 were inhibited by miR-370. Furthermore, the invasion and proliferation of human umbilical vein endothelial cells were promoted by miR-370 via inhibiting the expression of FOXO1. The results obtained in the present study demonstrated that miR-370 served an important role in regulating coronary atherosclerosis via targeting FOXO1. The present data also indicated that miR-370 may be a promising molecular target for treating coronary atherosclerosis.
\end{abstract}

\section{Introduction}

According to the investigation of the World Health Organization (2015), cardiovascular disease, a global health problem, is the leading cause of mortality globally $(1,2)$. In China, the number of patients with cardiovascular disease is projected to increase annually $(1,2)$. The tendency of development of cardiovascular disease in China is consistent with the global increase $(1,2)$. Atherosclerosis is a chronic disease that is induced by the accumulation of fibrous elements and lipids in the larger arteries and frequently causes severe problems,

Correspondence to: Dr Xin Chen, Department of Thoracic and Cardiovascular Surgery, Nanjing First Hospital, Nanjing Medical University, 68 Changle Road, Nanjing, Jiangsu 210006, P.R. China E-mail: xin_chen88@hotmail.com

Key words: coronary atherosclerosis, human umbilical vein endothelial cells, microRNA-370, Forkhead Box 1, apoptosis including blocking the aorta and aorta rupture bleeding (1-3). Atherosclerosis that occurs in the coronary artery is termed coronary atherosclerosis. Coronary atherosclerosis, particularly atherosclerosis of the external branch of the coronary artery, is the most common constrictive coronary artery disease (1-3). Numerous factors, including smoking, lack of exercise and dietary habits are associated with the production of coronary atherosclerosis (1-4). Due to the complicated mechanisms underlying cardiovascular disease, there are currently no successful treatments; therefore, there is a requirement to determine the possible mechanism underlying cardiovascular disease, and future studies should investigate the biomarkers of coronary atherosclerosis. These studies will provide data, for the early diagnosis and individual therapy of cardiovascular disease.

MicroRNAs (miRs) are defined as noncoding RNA molecules involved in regulating and controlling the posttranscription expression of target mRNAs $(5,6)$. They have complementary sequences in the 3 '-untranslated region (UTR) and 5'-UTR of target mRNAs and regulate the expression of protein-coding genes by causing mRNA cleavage (1). Previous studies have demonstrated that miRs can directly or indirectly regulate the expression of intercellular adhesion molecules and various inflammatory factors, including those involved in cell cycle progression, cell proliferation, lipid metabolism, endothelial cell function, angiogenesis, and plaque formation and rupture. These processes involve the regulation of vascular endothelial cells, vascular smooth muscle cells and macrophage function; therefore, the pathophysiology of atherosclerosis and cardiovascular disease are affected $(3,5,6)$. As for miRs in coronary atherosclerosis or other cardiovascular diseases, a number of studies have published their outcomes. For example, Jiang et al (7) demonstrated that miR is a signature and biomarker in chronic cardiovascular diseases. It has been reported by Hoekstra et al (8) that miR-370 is notably increased in PBMCs of patients with coronary atherosclerosis. In addition, Liu et al (9) revealed that plasma expression levels of miR-370 were significantly higher in patients with coronary artery disease compared with patients with non-coronary artery disease. Also, miR-370 was identified to be critical in the lipid metabolism, which is a potential biomarker for the diagnosis of coronary artery disease (7). However, the effect of miR-370 in critical cellular processes associated 
with coronary atherosclerosis remains unknown and requires further study.

\section{Materials and methods}

Chemicals and materials. Fetal bovine serum (FBS) was purchased from Gibco (Thermo Fisher Scientific, Inc., Waltham, MA, USA) and Dulbecco's modified Eagle's medium (DMEM) was purchased from Dalian Meilun Biology Technology Co., Ltd (Dalian, China). Smooth muscle cell medium (SMCM; cat. no. 1101; Sciencell Research Laboratories, Inc., San Diego, CA, USA) was also obtained. Antibodies of Forkhead Box 1 (FOXO1; cat. no. 2880), B-cell lymphoma 2 (Bcl-2)-associated X (Bax; cat. no. 5023), Bcl-2 (cat. no. 4223), cleaved-poly (ADP-ribose) polymerase (PARP; cat. no. 5625) and $\beta$-actin (cat. no. 4970) were purchased from Cell Signaling Technology, Inc. (Danvers, MA, USA). Antibodies for caspase 3 (cat. no. ab2302) were obtained from Abcam (Cambridge, MA, USA). Human umbilical vein endothelial cells HUVECs and 293 cells were obtained from the Type Culture Collection of the Chinese Academy Sciences (Shanghai, China). Reverse transcription-quantitative polymerase chain reaction (RT-qPCR)-associated chemicals were purchased from Thermo Fisher Scientific, Inc. The miR-370 mimics, miR-370 inhibitor, hsa-miR-370 mimics and miRNA negative control (miR-NC) were provided by Shanghai GenePharma Co., Ltd. (Shanghai, China). Western blot and gel analysis instruments were purchased from Bio-Rad Laboratories, Inc. (Hercules, CA, USA). All other reagents were of the highest purity and were commercially available from Shenyang LaiBo Science and Trade Co., Ltd (http://www.11467.com/shenyang/co/75880.htm, Shenyang, China).

Clinical samples. A total of 20 patients (10 male, 10 female; age, 40-60 years) with coronary atherosclerosis who required coronary artery bypass surgery, as confirmed by a coronary angiogram, and 20 healthy patients without any abnormal conditions in the coronary artery and heart, as confirmed by a coronary angiogram, were treated at Nanjing First Hospital, Nanjing Medical University (Nanjing, China). Patients with either of the following conditions were excluded: type 1 diabetes mellitus, autoimmune disease, malignancy, chronic or acute inflammatory disease, asthma, severe heart failure and renal and hepatic dysfunction. Clinical samples used in the present experiment were obtained from the Nanjing First Hospital, Nanjing Medical University from October 2016 to October 2017. Written informed consent was obtained in all cases. The study protocol was approved by the Ethics Committee at Nanjing First Hospital, Nanjing Medical University. PBMCs were obtained from these volunteers and separated using the Ficoll-Hypaque gradient separation technique (10). The samples were collected and immediately stored in a refrigerator at $-80^{\circ} \mathrm{C}$. The differential expression of miR-370 between patients with coronary atherosclerosis and healthy patients was detected using RT-qPCR. Simultaneously, the association between the expression of miR-370 and clinical information of patients was also analyzed.

Cell culture and cell transfection. HUVECs were cultivated in SMCM culture containing $10 \% \mathrm{FBS}$ in a $5 \% \mathrm{CO}_{2}$ incubator at $37^{\circ} \mathrm{C}$ with $70-80 \%$ humidity. The $293 \mathrm{~T}$ cells were cultivated in DMEM culture supplemented with $10 \% \mathrm{FBS}$ in a $5 \% \mathrm{CO}_{2}$ incubator at $37^{\circ} \mathrm{C}$ with $70-80 \%$ humidity. miR-370 mimics (5'-GCCUGCUGGGGUGGAACCUGGU-3'), miR-370 inhibitor (5'-CGGACGACCCCACCUUGGACCA-3') and miR-NC (5'-GCCAGCCGUUGUGGCAGAUGGU-3') were used.

HUVECs in the logarithmic phase were obtained and transfected with miR-370 mimics, miR-370 inhibitors and miR-NC with Lipofectamine 2000 (Invitrogen; Thermo Fisher Scientific, Inc.). The $10 \mathrm{nM}$ miR-370 mimics (11) were used for miR-370 overexpression and an equal quantity of miR-370 inhibitors was used for miR-370 downregulation. Simultaneously, miR-NC was used in the present study. miR-370 mimics and inhibitors are chemically modified small RNAs, and miR-370 mimics are double-stranded RNAs; however, miR-370 inhibitors are single-stranded RNAs (12). miR-370 was used to upregulate miR-370 activity and mimic endogenous miR-370. Additionally, miR-370 inhibitors were used to inhibit endogenous miR-370 molecules and empower miR-370 functional analysis by downregulating of miR-370. At $48 \mathrm{~h}$ post-transfection, total RNA were extracted with a TRIzol assay and analyzed with RT-qPCR to detect the ratio of transfection.

Cell proliferation assay [Cell counting kit-8 (CCK-8) assay]. HUVECs were selected for the cell proliferation assay. The cell proliferation value was measured by CCK-8 (Thermo Fisher Scientific, Inc.) at 0, 24, 48 and $72 \mathrm{~h}$ post-transfection. In brief, the cell viability of cells collected from different time points post-transfection was determined with CCK-8 in triplicate. CCK- 8 reagent $(10 \mu \mathrm{l})$ was added to $1 \times 10^{3}$ cells and incubated for $1 \mathrm{~h}$ at $37^{\circ} \mathrm{C}$. Subsequently, the absorbance of the resulting product was measured at $450 \mathrm{~nm}$ using a microplate reader (L-117; Thermo Fisher Scientific, Inc.).

miR analysis using RT-qPCR. HUVECs were cultured in 6 -well plates and harvested at $80 \%$ cell density. The total RNA were extracted according to the manufacturer's protocols using TRIzol reagent (Invitrogen; Thermo Fisher Scientific, Inc.). In brief, cells were centrifuged at 2,000 x $\mathrm{g}$ for $5 \mathrm{~min}$ at room temperature (RT). Subsequently, the supernatant was removed and the residue was combined with $1 \mathrm{ml}$ TRIzol. Following $5 \mathrm{~min}$ at RT, it was transferred into a new $1.5 \mathrm{ml}$ Eppendorf tube. Following the addition of $200 \mu \mathrm{l}$ chloroform, the mixture was placed at RT for $10 \mathrm{~min}$. Subsequently, the mixture was centrifuged at $12,000 \mathrm{x}$ g for $15 \mathrm{~min}$ at $4^{\circ} \mathrm{C}$. The upper supernatant was collected and combined with $200 \mu \mathrm{l}$ pre-cold isopropanol. Following an incubation for $10 \mathrm{~min}$ at $4^{\circ} \mathrm{C}$, the mixture was centrifuged at $12,000 \mathrm{x}$ for $12 \mathrm{~min}$ at $4^{\circ} \mathrm{C}$ and the supernatant was discarded. The residue was washed with $1 \mathrm{ml} 75 \%$ ethanol (prepared with fresh diethyl pyrocarbonate water). Subsequently, the suspension was centrifuged at $10,800 \mathrm{x}$ g for $5 \mathrm{~min}$ at $4^{\circ} \mathrm{C}$ twice, and then the supernatant was discarded. Once the total RNA was obtained, reverse transcription into cDNA was performed according to the manufacturer's protocols of the High-Capacity cDNA Reverse Transcription kit (Thermo Fisher Scientific, Inc.). U6 was selected as the internal control. The sequences of miR-370 and U6 were as follows: miR-370, forward 5'-TAGCCTGCT GGGGTGGAA-3' and reverse 5'-TATGGTTTTGACGAC 
TGTGTGAT-3'; and U6, forward 5'-ATTGGAACGATACAG AGAAGATT-3' and reverse 5'-GGAACGCTTCACGAATTT G-3'. In order to quantify the expression of $\mathrm{miR}$, the cycle quantification $\left(\mathrm{C}_{\mathrm{q}}\right)$ was determined using the TaqMan small RNA assay (Thermo Fisher Scientific, Inc.) with miR-specific primers, according to manufacturer's protocols. In brief, $7.6 \mu 1$ nuclease-free water and $10 \mu 1$ probe qPCR mix were added to $1.4 \mu \mathrm{l}$ cDNA. The TaqMan small RNA assay primer of has-miR-9-5p was used. RT-qPCR reactions were conducted with a QuantStudio 5 Real-Time PCR System (Thermo Fisher Scientific, Inc.). Samples were run in triple for each experiment. PCR cycling procedures were as follows: $95^{\circ} \mathrm{C}$ for $5 \mathrm{~min}$, followed by 35 cycles of $95^{\circ} \mathrm{C}$ for $40 \mathrm{sec}, 60^{\circ} \mathrm{C}$ for $40 \mathrm{sec}, 72^{\circ} \mathrm{C}$ for $40 \mathrm{sec}$ and $72^{\circ} \mathrm{C}$ for $10 \mathrm{~min}$.

The expression of miR-370 in PBMCs was also determined with RT-qPCR. The method of sample preparation was performed as mentioned above.

Wound healing assay. The influence of miR-370 on the wound healing of HUVECs was measured using a wound healing assay. In brief, HUVECs were cultured in 6-well plates and transfected according to the aforementioned method. At $24 \mathrm{~h}$ post-transfection, the ratio of transfection was $\sim 90 \%$. The vertically lineation was scratched on the cell culture plate with a $200 \mu$ l pipette tip. Following washing with PBS three times, cells were cultured in SMCM without serum for $24 \mathrm{~h}$ at $37^{\circ} \mathrm{C}$. The width of the scratch at different time points (0 and $24 \mathrm{~h})$ was recorded under a light microscope (magnification, x200). Samples were run in triplicate for each experiment.

Matrigel invasion assay. HUVEC invasion was conducted using BioCoat Matrigel Invasion Chambers (cat. no. 354480; Corning Incorporated, Corning, NY, USA), according to the manufacturer's protocols. The upper chamber was pre-coated with $100 \mu \mathrm{l}$ Matrigel and exposed to ultra violet light for 2 h. DMEM medium containing with $10 \%$ FBS $(500 \mu \mathrm{l})$ was added to the lower wells. Following transfection with miR-370 mimics, the HUVECs, in serum-free SMCM medium, were added to the upper well $\left(4 \times 10^{4}\right.$ cells $\left./ \mathrm{ml}\right)$. Subsequently, the cells were incubated at $37^{\circ} \mathrm{C}$ for $24 \mathrm{~h}$. Following this, the cells on the surface of the upper chamber membrane were discarded with cotton swabs. The cells underside of the membrane were fixed in $100 \%$ methanol and stained with a solution containing $50 \%$ isopropanol, $1 \%$ formic acid and $0.5 \%$ crystal violet for $20 \mathrm{~min}$ at RT. Subsequently, the cells were counted under a light microscope (views were randomly selected, at least 5 views/well; magnification, x200). Results were presented by mean \pm standard deviation (SD). Samples were run in sextuple for each experiment.

Cell apoptosis assay. The collected HUVECs at $48 \mathrm{~h}$ post-transfection were washed two times with cold PBS, centrifuged at $1,000 \mathrm{x}$ g for $5 \mathrm{~min}$ at $4^{\circ} \mathrm{C}$ and then the supernatant was discarded. The residue was resuspended in $100 \mu \mathrm{l}$ binding buffer (Thermo Fisher Scientific, Inc.). Subsequently, $4 \mu$ l Annexin V-fluorescein isothiocyanate and $3 \mu \mathrm{l}$ propidium iodide (Thermo Fisher Scientific, Inc.) were added in the dark. Following incubating for $15 \mathrm{~min}$ at RT, $200 \mu \mathrm{l}$ binding buffer was added and measured using a flow cytometer (BD Biosciences, San Jose, CA, USA). The same experiments were performed in triplicate.
The dual luciferase reporter system assay. A total of three types of internet-based bioinformatics online software, TargetScan 6.0 (http://www.targetscan.org/vert_71/), miRanda (http://www.microrna.org/microrna/home.do) and miRbase (http://www.mirbase.org/), were used to search the specific targets between miR-370 and the 3'-untranslated region (UTR) of FOXO1. Based on the complementarity nucleotide sequence to the 3'-UTR region of FOXO1 mRNA, the specific targets were recognized. According to the information recorded on microRNA.org, miR expression data were obtained. The target gene sequence and the mutation gene sequence were synthesized by Sangon Biotech Co., Ltd. (Shanghai, China). The dual luciferase reporter system assay kit was provided by Promega Corporation (Madison, WI, USA) and contained the luciferase reporter plasmid. The gene sequence was bound with the carrier psiCHECK-2. Gene sequences were digested using Xho I and Not I enzymes and the sequences were authenticated by electrophoresis and sequencing. 293 cells in the exponential phase were selected for the present assay. The psiCHECK2-FOXO1-wild type (WT) and psiCHECK2-FOXO1-mutation (MT) were treated with hsa-miR-370 mimics separately. Lipofectamine 2000 was used in the transfection procedure according to the manufacturer's protocols. Following incubation in $5 \% \mathrm{CO}_{2}$ for $24 \mathrm{~h}$ at $37^{\circ} \mathrm{C}$, the medium was replaced with fresh SMCM medium. Subsequently, the 293T cells were transfected for 48 h. Three samples were contained in each group. Following transfection, the 293 cells in WT and MT groups were digested using tritonX-100. The lysate was treated with Firefly luciferase and Renilla luciferase buffer solution and their relative substrate separately. Firefly luciferase was used as the internal reference of psiCHECK-2 and the expression of the carrier psiCHECK-2+miR-NC was used as the control. Luciferase activities were determined with a dual-luciferase assay kit $48 \mathrm{~h}$ following transfection.

Western blot analysis. HUVECs were lysed with radioimmunoprecipitation assay buffer containing protease inhibitor cocktail (Dalian Meilun Biotech Co., Ltd., Dalian, China). Subsequently, the lysate was centrifuged for $10 \mathrm{~min}$ at $10,800 \mathrm{x} \mathrm{g}$ at $4^{\circ} \mathrm{C}$. The supernatant was obtained for further analysis. Total protein was measured using a Pierce BCA protein assay kit (Thermo Fisher Scientific, Inc). Equal amounts of total protein $(40 \mu \mathrm{g})$ were subjected to SDS-PAGE (10\% gels) and transferred onto polyvinylidene fluoride membranes (PVDF; Dalian Meilun Biotech Co., Ltd.) with a wet transmembrane device (Thermo Fisher Scientific, Inc.). The following primary antibodies were used: Anti-FOXO1 (1:1,000 dilution), anti-Bax (1:1,000 dilution), anti-Bcl-2 (1:1,000 dilution), anti-cleaved PARP (1:1,000 dilution) anti-active caspase 3 (1:1,000 dilution) and anti- $\beta$-actin $(1: 1,000)$. Following blocking with $5 \%$ non-fat milk at RT for $1 \mathrm{~h}$, the membranes were incubated overnight with primary antibodies and incubated with the appropriate horseradish peroxidase (HRP)-conjugated secondary antibodies (HRP-anti-rabbit, cat. no. 0208, 1:5,000 dilution; HRP-anti-goat, cat. no. 0216, 1:5,000 dilution; both Beyotime Institute of Biotechnology, Shanghai, China) for $2 \mathrm{~h}$ at RT. Subsequently, the PVDF membranes were incubated with enhanced chemiluminescence reagent (Santa Cruz Biotechnology, Inc., Dallas, TX, USA), in order to develop the 

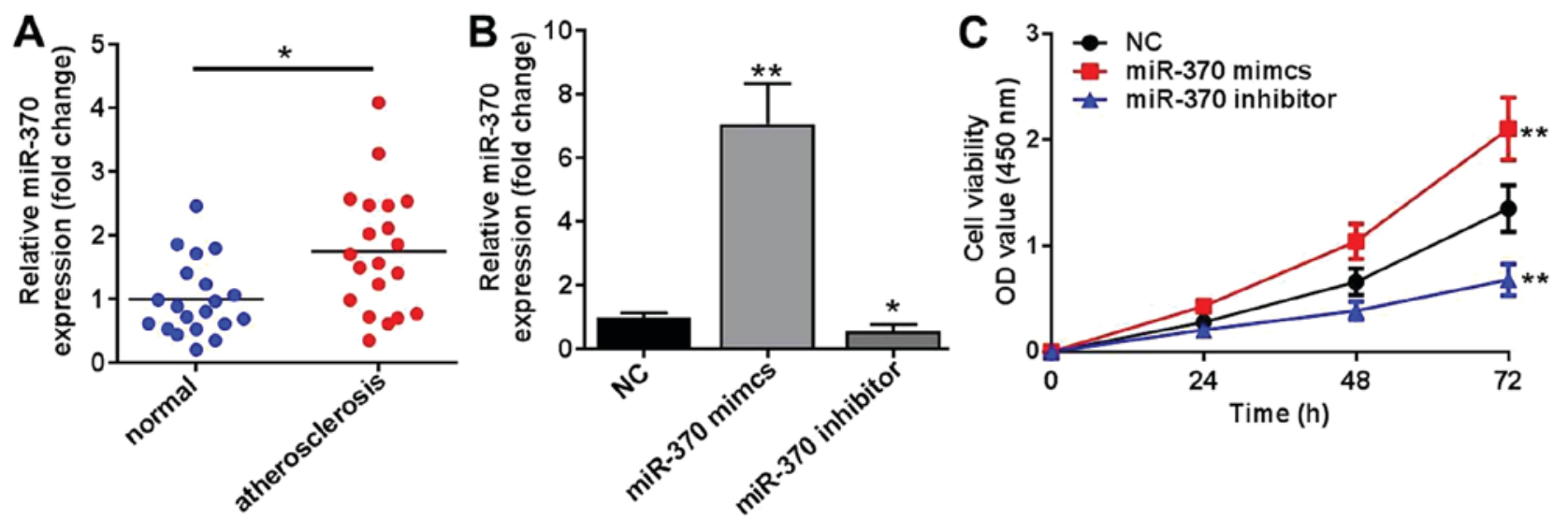

Figure 1. miR-370 enhances the activity of HUVEC proliferation. (A) Relative miR-370 expression in PBMCs of healthy patients and patients with atherosclerosis ( $\mathrm{n}=20$ in each group). ${ }^{*} \mathrm{P}<0.05$ as indicated. (B) Relative miR-370 expression in HUVECs following transfection with miR-370 mimics and inhibitors. (C) Effect of miR-370 on HUVEC proliferation following transfection with miR-370 mimics and inhibitors. Relative miR-370 expression was measured using reverse transcription-quantitative polymerase chain reaction. The cell proliferation assay was performed using Cell Counting kit-8. miR-370 mimics and inhibitors were transfected into HUVECs using Lipofectamine 2000. Values were presented as the mean \pm standard deviation. ${ }^{*} \mathrm{P}<0.05$ and ${ }^{* * *} \mathrm{P}<0.05$ vs. NC group. NC, negative control; miR, microRNA; PBMC, peripheral blood mononuclear cells; HUVEC, human umbilical vein endothelial cells.

blots. All values were normalized to those of $\beta$-actin. Protein densitometry was determined using Image-Pro Plus 6.0 software (Media Cybernetics, Inc., Rockville, MD, USA).

Statistical analysis. Values were expressed as the mean \pm SD. All experiments were conducted a minimum of three times. All statistical analyses were calculated using SPSS 19.0 software (IBM Corp., Armonk, NY, USA). One-way analysis of variance followed by Dunnett's post hoc test was used to compare the differences between multiple groups. Statistical differences between two groups were determined using the Student's t-test. $\mathrm{P}<0.05$ was considered to indicate a statistically significant difference.

\section{Results}

miR-370 enhances the cell proliferation of HUVECs. Compared with healthy patients, the expression levels of miR-370 in PBMCs of patients with atherosclerosis were significantly increased, which indicated that the abnormal expression of miR-370 was associated with atherosclerosis (Fig. 1A). Following transfection with miR-370 mimics, the expression levels of miR-370 in HUVECs were significantly increased compared with the NC group (Fig. 1B); however, the expression levels of miR-370 in HUVECs were significantly reduced compared with the NC group, following transfection with miR-370 inhibitors (Fig. 1C). These results demonstrated that the transfection model was successful. Following transfection with miR-370 mimics or inhibitors, HUVEC proliferation was affected in two different directions. Accompanied with the enhanced expression of miR-370 induced by transfection with miR-370 mimics, HUVEC proliferation was significantly increased compared with the NC group $(\mathrm{P}<0.01$; Fig. 1C). miR-370 enhanced the cell proliferation, which was also confirmed by the decreased cell viability in the miR-370 inhibitor group compared with the NC group (Fig. 1C).

miR-370 promotes cell migration and invasion. As depicted in Fig. 2A, the distance in the NC, miR-370 mimics and miR-370 inhibitor groups was similar at $0 \mathrm{~h}$; however, the distance in the miR-370 mimics group was reduced at $24 \mathrm{~h}$ compared with the NC group. According to the results observed in the wound healing assay, overexpression of miR-370 significantly promoted the activity of cell migration compared with the NC group (Fig. 2B). The invasion capacity of HUVECs, which were transfected with the miR-370 mimics, was evaluated by performing the Matrigel assay. As indicated in Fig. 2C and D, upregulation of miR-370 with miR-370 mimics significantly promoted cell migration compared with the NC group. Notably, miR-370 inhibitors did not affect cell migration and invasion (Fig. 2A-D).

Downregulation of miR-370 promotes cell apoptosis. As depicted in Fig. 3A, a notable number of apoptotic cells were observed in HUVECs transfected with miR-370 inhibitors. Based on the results obtained with flow cytometry, the apoptosis rate was calculated and depicted in Fig. 3B. The apoptotic rate in the NC and miR-370 mimics groups were low. According to the results depicted in Fig. 1B, the expression of miR-370 was significantly reduced following transfection with miR-370 inhibitors; however, the apoptotic rate of HUVECs in the miR-370 inhibitor group was significantly increased compared with the NC group. Collectively, the results demonstrated that downregulation of miR-370 promoted cell apoptosis. Notably, miR-370 mimics could not induce HUVEC apoptosis.

miR-370 is a regulator of FOXO1. The miRNA sequence in the bioinformatics platforms was used to search for potential targets, which were weighted and a list of the optimal potential targets are generated. According to the three most commonly used bioinformatics prediction tools (TargetScan 6.0, miRanda and miRbase), protein FOXO-1 was projected as the most possible target of miR-370; the specific combined targets of miR-370 on FOXO1 gene was focused from 46-53 of FOXO1 3'-UTR (Fig. 4A); therefore, FOXO1 was indicated as the target gene of miR-370. In order to confirm the result, the dual luciferase reporter assay was performed. 293T cells, the most 
A
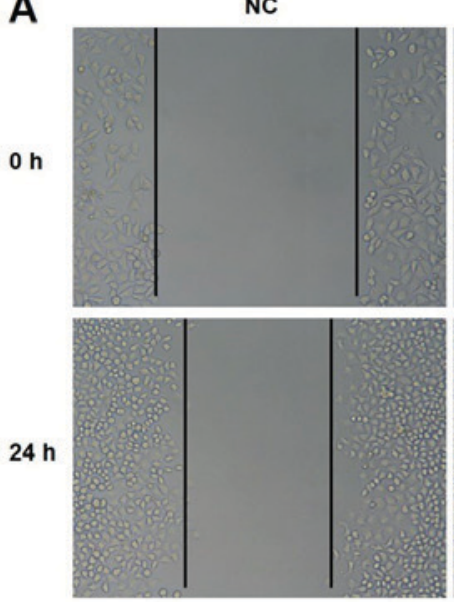

C

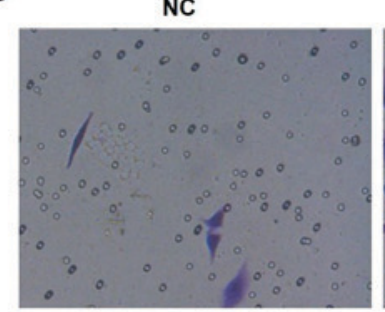

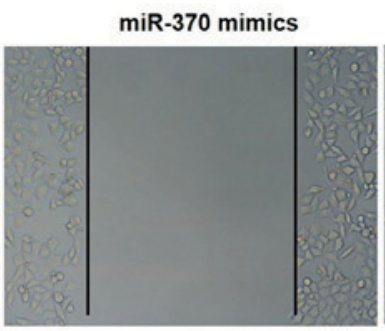

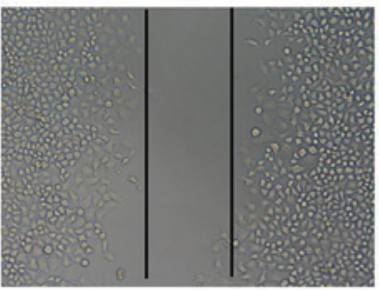

miR-370 mimics

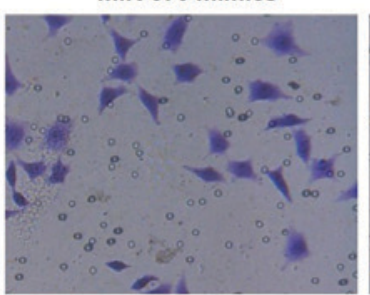

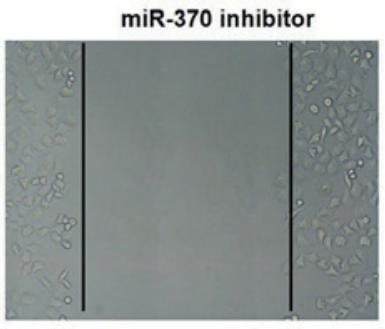

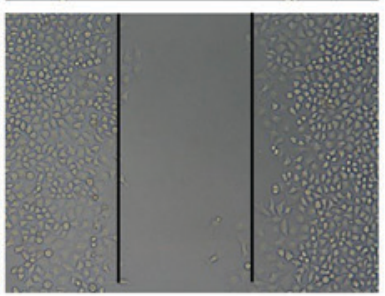

miR-370 inhibitors

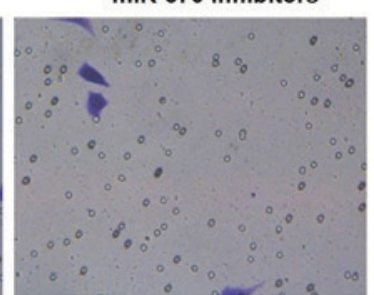

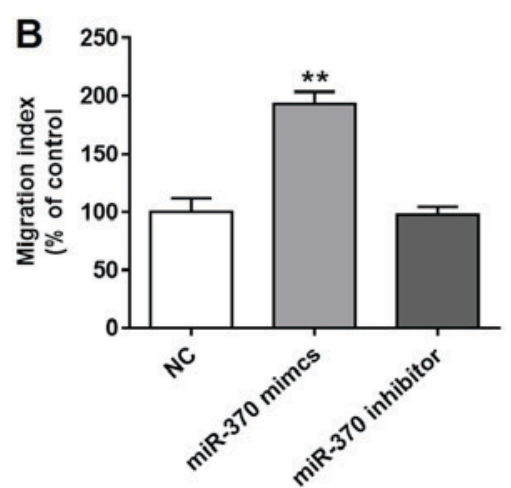

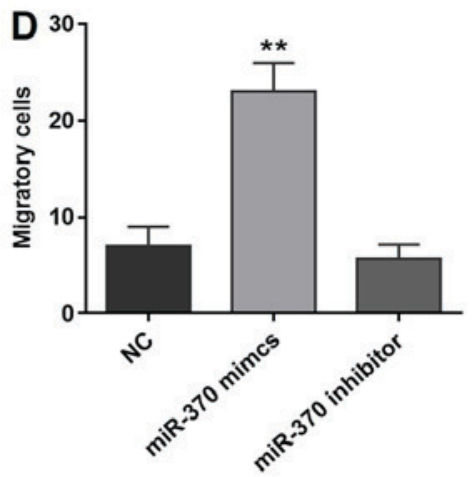

Figure 2. miR-370 promotes cell migration and invasion. (A) Effect of miR-370 on cell migration with a wound healing assay (magnification, x200). (B) Effect of miR-370 on cell invasion with a Matrigel invasion assay. Invaded cells were counted under a light microscope (views were randomly selected, at least five views/well; magnification, x200). (C) Effect of miR-370 on cell invasion with a Matrigel invasion assay. Invaded cells were counted under a light microscope (views were randomly selected, at least five views/well; magnification, x200). (D) Migratory cells in each group were quantified. Values were presented as the mean \pm standard deviation. ${ }^{* *} \mathrm{P}<0.05$ vs. NC group. NC, negative control; miR, microRNA.

A
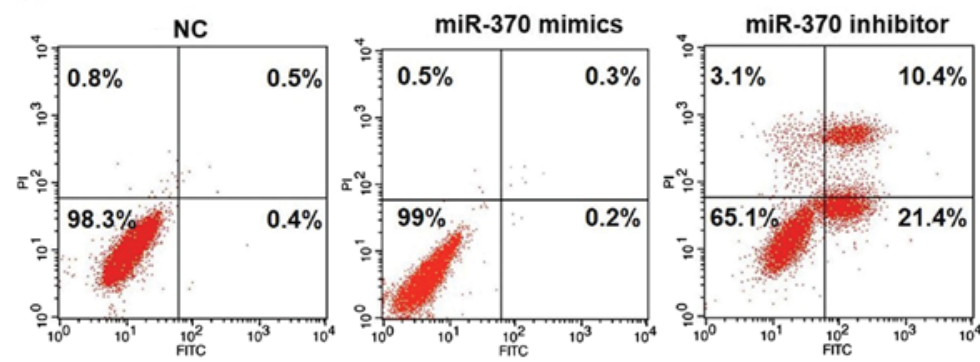

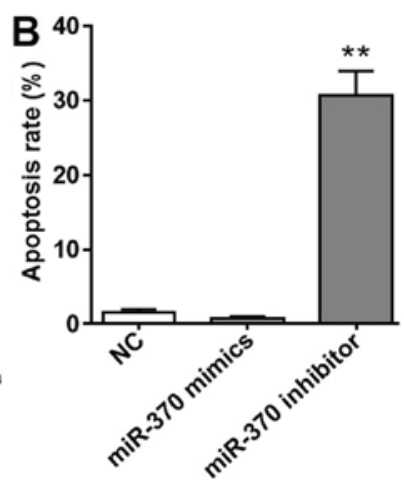

Figure 3. Downregulation of miR-370 promotes cell apoptosis. (A) Effect of downregulation of miR-370 on cell apoptosis by Annexin V and propidium iodide staining using flow cytometry. (B) Apoptosis rate of HUVECs was determined following transfection with miR-370 inhibitors. Values were presented as the mean \pm standard deviation. ${ }^{* *} \mathrm{P}<0.05$ vs. NC group. NC, negative control; HUVEC, human umbilical vein endothelial cells; miR, microRNA.

commonly used tool in this assay, were selected. The luciferase activity was significantly reduced following transfection with psiCHECK-2-FOXO1-WT and miR-370 mimics compared with the NC group (Fig. 4B); however, the luciferase activity was not significantly affected when the specific target was mutated (Fig. 4B). Therefore, miR-370 was indicated as a vital regulator of the FOXO1 gene. Furthermore, the western blot analysis result of the FOXO1 protein performed on HUVECs also supported this conclusion. As depicted in Fig. 4C and D, the protein expression levels of FOXO1 in HUVECs following transfection with miR-370 mimics was significantly reduced compared with the NC group. However, HUVECs in the presence of miR-370 inhibitors exhibited increased expression levels of FOXO1 protein compared with the NC group.

miR-370 regulates cell apoptosis by affecting relative target proteins. FOXO1, as a transcription factor, serves a vital role in numerous crucial cellular processes, including cell apoptosis and cell cycle progression (13). FOXO1 is considered to be involved in the process of inducing cell apoptosis through its transcription of a number of genes in the apoptotic pathway and its location in the cell nucleus $(13,14)$. A 

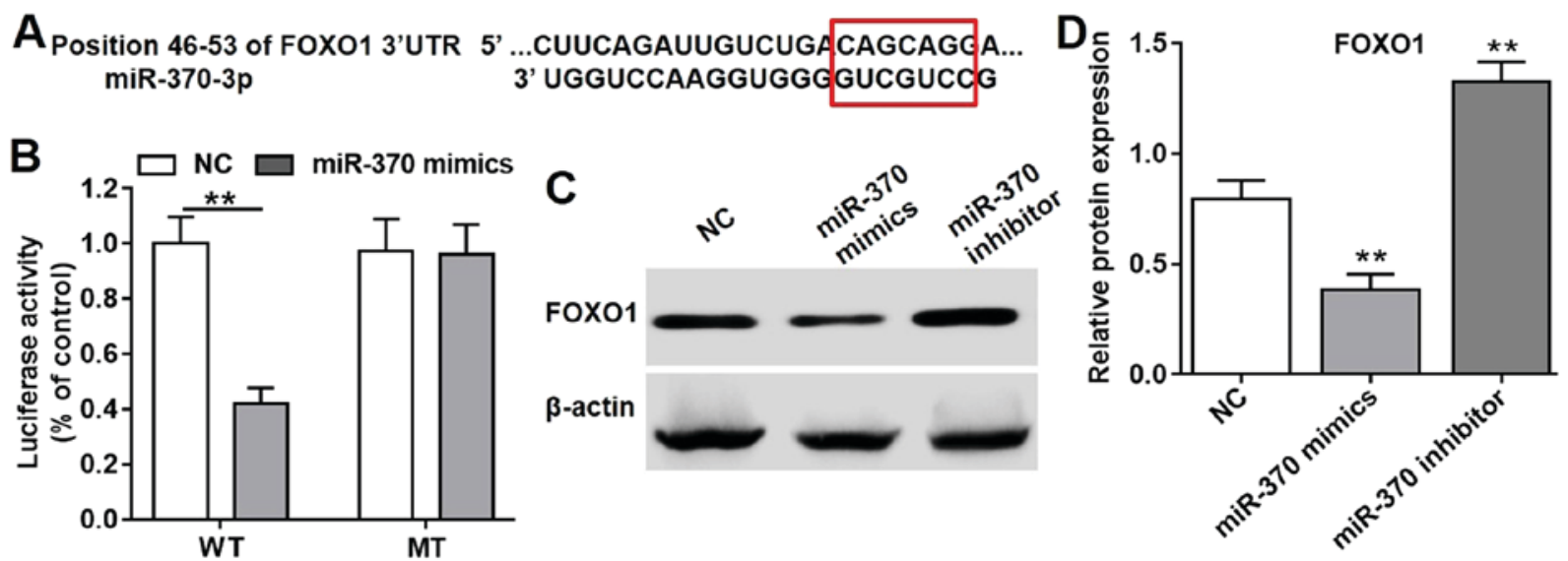

Figure 4. miR-370 is a regulator of FOXO1. (A) The specific combined position between FOXO1 3'-UTR and miR-370 based on the results of bioinformatics tools. (B) Luciferase activity was measured using the dual luciferase reporter assay. 293T cells were selected and used in the present assay. Cells in the WT group were treated with psiCHECK-2-FOXO1-WT+miR-NC and psiCHECK-2-FOXO1-WT+miR-370 mimics separately. Cells in the MT group were treated with psiCHECK-2-FOXO1-MT+miR-NC and psiCHECK-2-FOXO1-MT+miR-370 mimics separately. "* P $<0.01$ as indicated. (C) Effect of miR-370 on FOXO1 protein expression in HUVECs following transfection with miR-370 mimics and miR-370 inhibitors. The expression levels of FOXO1 were detected using western blot analysis. (D) Relative protein expression levels of FOXO1 in HUVECs quantified by Image pro plus 6.0. Values were presented as the mean \pm standard deviation. ${ }^{* *} \mathrm{P}<0.05$ vs. NC group. NC, negative control; WT, wild type; MT, mutation type; HUVEC, human umbilical vein endothelial cells; miR, microRNA; FOXO1, Forkhead Box 1.
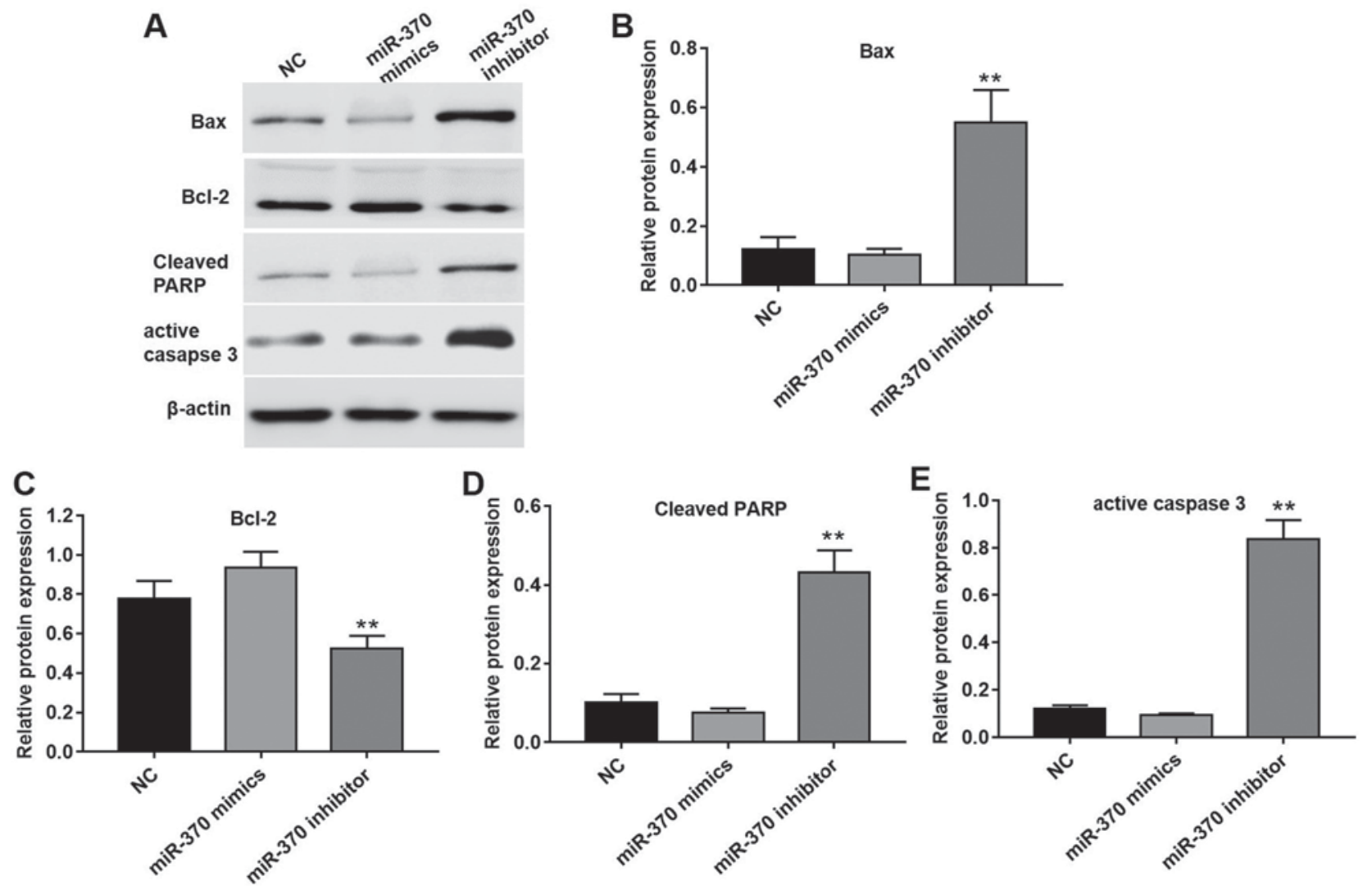

Figure 5. miR-370 regulated cell apoptosis by affecting relative target proteins. (A) Effect of overexpression and downregulation of miR-370 on apoptotic proteins, including Bax, Bcl-2, cleaved-PARP and active caspase 3. Effect of overexpression and downregulation of miR-370 on apoptotic relative proteins (B) Bax, (C) Bcl-2, (D) cleaved-PARP and (E) active caspase 3 in HUVECs quantified by Image pro plus 6.0. Values were presented as the mean \pm standard deviation. ${ }^{* *} \mathrm{P}<0.05$ vs. NC group. NC, negative control; miR, microRNA; Bcl-2, B-cell lymphoma-2; Bax, Bcl-2-associated X; HUVEC, human umbilical vein endothelial cells; PARP, poly(ADP-ribose) polymerase.

total of four apoptotic proteins, Bax, Bcl-2, cleaved-PARP and active caspase 3 , were selected to evaluate the association between miR-370 and cell apoptosis (Fig. 5). Following transfection with miR-370 mimics, protein expression levels of pro-apoptotic proteins, including Bax, cleaved-PARP and active caspase 3 , were reduced compared with the $\mathrm{NC}$ group (Fig. 5A and B). However, the expression levels of Bcl-2, a type of inhibitor of apoptotic proteins, was significantly decreased 
following transfection with miR-370 inhibitors compared with the NC group (Fig. 5A and C). In addition, miR-370 inhibitors significantly increased the expression levels of Bax, cleaved-PARP and active caspase 3 in HUVECs following transfection (Fig. 5A, B, D and E).

\section{Discussion}

miR-370 is considered to be a key miR in lipid metabolism that downregulates the expression of the carnitine palmitoyl transferase $1 \alpha$ gene, which controls fatty acid oxidation (15). Liu et al (9) revealed that plasma expression levels of miR-370 were significantly higher in the patients with coronary artery disease compared with those without coronary artery disease (9). Consistent with a previous report (9), the expression levels of miR-370 in the PBMCs of patients with atherosclerosis were significantly increased. In addition, it has been reported that miR-370 expression levels are potential diagnostic tools for discriminating coronary artery disease to facilitate with the management of patient care (9). However, to the best of our knowledge, there is no report on the function of miR-370 in atherosclerosis.

In the present study, the function and target gene of miR-370 in HUVECs were investigated. HUVECs are the most commonly used model for the study of coronary atherosclerosis, as the proliferation and migration of endothelial cells (ECs) are vital processes involved in atherosclerosis (2). The present study results suggested that overexpression of miR-370 enhanced cell migration and invasion. The enhanced invasion of assorted exogenous cells across a stiffened extracellular matrix and an increase in the migratory behavior of resident cells are considered to characterize atherosclerosis (16). For example, as an RNA aptamer, Apt-alphavbeta3 has been identified to reduce platelet-derived growth factor-stimulated tube formation and to increase HUVEC apoptosis through inhibition of focal adhesion kinase phosphorylation pathway. In addition, human endothelial cell proliferation and survival were significantly inhibited by Apt-alphavbeta3, which in turn resulted in reduced angiogenesis (17). Similarly, the present study indicated that downregulation of miR-370 promoted cell apoptosis.

In order to further investigate the underlying molecular target involved in miR-370-mediated atherosclerosis, three tools, namely TargetScan 6.0, miRanda and miRbase, were used. According to the result of these three tools, protein FOXO-1 was considered to be the possible target of miR-370. FOXO-1 was selected in the present study to investigate its association with miR-370. The dual luciferase reporter system assay suggested that miR-370 could bind to the 3'-UTR of FOXO1 mRNA, which resulted in the inhibition of FOXO1 expression.

FOXOs have been confirmed to be essential regulators of cellular homeostasis, including oxidative stress response and redox signaling, lipid metabolism, apoptosis and cell cycle progression $(18,19)$. FOXO1 has been considered to be important in different types of cellular action $(18,19)$. Deng et al $(20)$ demonstrated that FOXO1 served an important role in vascular smooth muscle cell calcification and the ubiquitination of PTEN/Akt-modulated Runt-associated transcription factor 2. Li et al (21) demonstrated that FOXO1 and the pro-apoptotic protein Bcl-2 like 11 (Bim) served vital roles in regulating endothelial cell apoptosis induced by oxidative stress; however, endothelial cell apoptosis caused by oxidative stress is an early event in the development of atherosclerosis. Furthermore, miR-370 has been reported to be effective in regulating the proliferation of human prostate cancer cells through directly inhibiting the tumor suppressor FOXO1 $(12,18,19)$. According to the present results, miR-370 was a specific regulator of FOXO1, which was consistent with previous reports $(12,18,19)$.

Apoptosis has critical role in maintaining tissue homeostasis and eliminating damaged cells, and has been confirmed to be a programmed cell suicide mechanism (21). The Bcl-2 family and caspases are the two major regulators of apoptosis (21). According to reports published previously, FOXO1 is inhibited by the phosphorylation of Akt, while the downstream protein Bim is activated by the phosphorylation of FOXO1 (18-21). Additionally, Bim can directly activate the pro-apoptotic protein Bax, which is downstream of Bim and inhibits the anti-apoptotic protein Bcl-2 (21). Furthermore, PARP is inhibited by caspase 3 and cleaved PARP is an important protein in promoting cell apoptosis (22). These proteins are all involved in the mitochondrial apoptotic pathways (22).

Collectively, as a specific regulator of FOXO1, the present findings suggested that miR-370 serves a vital role in coronary atherosclerosis. Overexpression of miR-370 inhibited the expression of FOXO1. Furthermore, Bax, Bcl-2 and cleaved PARP expression levels were affected in the process of cell apoptosis; therefore, miR-370 has the potential to be used as a biomarker in identifying coronary atherosclerosis and may be used in treatment to improve coronary atherosclerosis. Further research on the function between these markers in vivo will provide an essential insight into basic and clinical processes. However, many miRNAs may be associated with atherosclerotic disease. Thus, the assessment of other miRNAs and their functional effects in atherosclerosis is required, which may offer a potential target for coronary artery disease therapy.

\section{Acknowledgements}

Not applicable.

\section{Funding}

The present study was supported by the Jiangsu Provincial Special Program of Medical Science (grant. no. BE2015612).

\section{Availability of data and materials}

The datasets used and/or analyzed during the current study are available from the corresponding author on reasonable request.

\section{Authors' contributions}

XS performed experiments, analyzed data and was the major contributor in developing the manuscript. XC collected tissues, interpreted the patient data and reviewed the final version of the manuscript.

\section{Ethics approval and consent to participate}

Ethical approval for the present study was received from Nanjing First Hospital, Nanjing Medical University. All 
patients provided written informed consent to participate in the study.

\section{Patient consent for publication}

All patients provided written informed consent for the publication of all associated information.

\section{Competing interests}

The authors declare that they have no competing interests.

\section{References}

1. Ambros V: The functions of animal microRNAs. Nature 431: 350-355, 2004

2. Xu Z, Han Y, Liu J, Jiang F, Hu H, Wang Y, Liu Q, Gong Y and Li X: MiR-135b-5p and MiR-499a-3p promote cell proliferation and migration in atherosclerosis by directly targeting MEF2C. Sci Rep 5: 12276, 2015.

3. Ndrepepa G, Colleran R and Kastrati A: Gamma-glutamyl transferase and the risk of atherosclerosis and coronary heart disease. Clin Chim Acta 476: 130-138, 2018

4. Zhang H, Jia K, Sun D and Yang M: Protective effect of HSP27 in atherosclerosis and coronary heart disease by inhibiting reactive oxygen species. J Cell Biochem: Dec 12, 2017 (Epub ahead of print).

5. Zhu N, Zhang D, Chen S, Liu X, Lin L, Huang X, Guo Z, Liu J, Wang Y, Yuan W and Qin Y: Endothelial enriched microRNAs regulate angiotensin II-induced endothelial inflammation and migration. Atherosclerosis 215: 286-293, 2011.

6. Quan X, Ji Y, Zhang C, Guo X, Zhang Y, Jia S, Ma W, Fan Y and Wang C: Circulating MiR-146a may be a potential biomarker of coronary heart disease in patients with subclinical hypothyroidism. Cell Physiol Biochem 45: 226-236, 2018.

7. Jiang Y, Wang HY, Li Y, Guo SH,Zhang L and Cai JH: Peripheral blood miRNAs as a biomarker for chronic cardiovascular diseases. Sci Rep 4: 5026, 2014.

8. Hoekstra M, van der Lans CA, Halvorsen B, Gullestad L, Kuiper J, Aukrust P, van Berkel TJ and Biessen EA: The peripheral blood mononuclear cell microRNA signature of coronary artery disease. Biochem Biophys Res Commun 394: 792-797, 2010.

9. Liu H, Yang N, Fei Z, Qiu J, Ma D, Liu X, Cai G and Li S: Analysis of plasma miR-208a and miR-370 expression levels for early diagnosis of coronary artery disease. Biomed Rep 5: 332-336, 2016
10. Märkl B, Wilhelms N, Anthuber M, Schenkirsch G, Schlimok G and Oruzio D: Circulating cytokeratin-positive cells and tumor budding in colorectal cancer. World J Clin Oncol 7: 433-440, 2016

11. Zeng L, Chen Y, Wang Y, Yu LR, Knox B, Chen J, Shi T, Chen S, Ren Z, Guo L, et al: MicroRNA hsa-miR-370-3p suppresses the expression and induction of CYP2D6 by facilitating mRNA degradation. Biochem Pharmacol 140: 139-149, 2017.

12. Wu Z, Sun H, Zeng W, He J and Mao X: Upregulation of MircoRNA-370 induces proliferation in human prostate cancer cells by downregulating the transcription factor FOXO1. PLoS One 7: e45825, 2012.

13. Xing YQ, Li A, Yang Y, Li XX, Zhang LN and Guo HC: The regulation of FOXO1 and its role in disease progression. Life Sci 193: 124-131, 2018.

14. Pan CW, Jin X, Zhao Y, Pan Y, Yang J, Karnes RJ,ZZhang J, Wang L and Huang H: AKT-phosphorylated FOXO1 suppresses ERK activation and chemoresistance by disrupting IQGAP1-MAPK interaction. EMBO J 36: 995-1010, 2017.

15. Iliopoulos D, Drosatos K, Hiyama Y, Goldberg IJ and Zannis VI: MicroRNA-370 controls the expression of microRNA-122 and Cptlalpha and affects lipid metabolism. J Lipid Res 51: 1513-1523, 2010.

16. Kai F, Laklai H and Weaver VM: Force Matters: Biomechanical regulation of cell invasion and migration in disease. Trends Cell Biol 26: 486-497, 2016.

17. Mi J, Zhang X, Giangrande PH, McNamara JO II, Nimjee SM, Sarraf-Yazdi S, Sullenger BA and Clary BM: Targeted inhibition of alphavbeta3 integrin with an RNA aptamer impairs endothelial cell growth and survival. Biochem Biophys Res Commun 338: 956-963, 2005 .

18. Berry E, Hardt JL, Clardy J, Lurain JR and Kim JJ: Induction of apoptosis in endometrial cancer cells by psammaplysene A involves FOXO1. Gynecol Oncol 112: 331-336, 2009.

19. Tsuchiya K and Ogawa Y: Forkhead box class O family member proteins: The biology and pathophysiological roles in diabetes. J Diabetes Investig 8: 726-734, 2017.

20. Deng L, Huang L, Sun Y, Heath JM, Wu H and Chen Y: Inhibition of FOXO1/3 promotes vascular calcification. Arterioscler Thromb Vasc Biol 35: 175-183, 2015.

21. Li Y, Ren M, Wang X, Cui X, Zhao H, Zhao C, Zhou J, Guo Y, $\mathrm{Hu}$ Y, Yan C, et al: Glutaredoxin 1 mediates the protective effect of steady laminar flow on endothelial cells against oxidative stress-induced apoptosis via inhibiting Bim. Sci Rep 7: 15539, 2017.

22. Zhu C, Zhu Q, Wu Z, Yin Y, Kang D, Lu S and Liu P: Isorhapontigenin induced cell growth inhibition and apoptosis by targeting EGFR-related pathways in prostate cancer. J Cell Physiol 233: 1104-1119, 2018.

c) (i) $\ominus$ This work is licensed under a Creative Commons (c) Attribution-NonCommercial-NoDerivatives 4.0 International (CC BY-NC-ND 4.0) License. 